\title{
Submillisecond electron spin relaxation in InP quantum dots
}

\author{
Michio Ikezawa, Bipul Pal, and Yasuaki Masumoto \\ Institute of Physics, University of Tsukuba, Tsukuba 305-8571, Japan \\ Ivan V. Ignatiev* and Sergey Yu. Verbin \\ Institute of Physics, University of Tsukuba, Tsukuba 305-8571, Japan \\ and Institute of Physics, St.-Petersburg State University, St.-Petersburg, 198504, Russia \\ Il'ya Ya. Gerlovin \\ Vavilov State Optical Institute, St.-Petersburg, 190034, Russia \\ (Received 12 July 2005; published 3 October 2005)
}

\begin{abstract}
Light-induced orientation of electron spins in the negatively charged InP quantum dots is found to persist longer than $100 \mu \mathrm{s}$. We have proved experimentally that the long-lived orientation is due to slow relaxation of the electron spins rather than to the dynamic nuclear polarization effects.

DOI: $10.1103 /$ PhysRevB.72.153302

PACS number(s): 78.67.Hc, 72.25.Fe, 73.21.La, 78.47.+p
\end{abstract}

In recent years, the long-lived spin orientation in semiconductor structures attracts increasing attention of researchers as a promising way of quantum information processing and storage. ${ }^{1}$ The structures with quantum dots (QDs) in which the main processes of spin relaxation are suppressed due to confinement of the carrier are particularly advantageous in this respect. According to the theoretical estimates, ${ }^{2}$ the electron spin lifetime in QDs can lie in the range of milliseconds or even seconds. This theoretical prediction has stimulated the studies of spin relaxation in low-dimensional structures. $^{3-8}$ The most exciting experimental results achieved in this field were the electron spin relaxation time $T_{1} \geqslant 50 \mu \mathrm{s}$, observed for the one-electron QD (Ref. 5) and $T_{1} \sim 20 \mathrm{~ms}$ observed for the InGaAs self-assembled QDs. ${ }^{7,8}$ At the same time, a fundamental question arises associated with the origin of so long-lived spin polarization. Along with suppression of main spin relaxation processes in QDs, predicted theoretically, ${ }^{2}$ persistence of the spin polarization can result from dynamic nuclear polarization ${ }^{10-13}$ capable to conserve the electron spin for a long time. It should be particularly emphasized that the dynamic nuclear polarization is a very slow process which cannot realize fast recording of information. The role of nuclear polarization has not been discussed in Refs. 5, 7, and 8 and the question about origin of the long-lived spin orientation in semiconductor QDs is open so far.

In this paper, we present the experimental results showing that relaxation of the electron spin in InP QDs is indeed very slow and the lifetime of real spin memory in this system can exceed $100 \mu \mathrm{s}$. On the analysis of the experimental data we came to conclusion that so long-lived spin memory is not associated with the nuclear polarization. To study the electron spin memory, we used a modification of the known pump-probe technique with detection of the polarized photoluminescence signal (PL pump probe).,6,9 The method involves detection of the effect of a circularly polarized pump on polarization of the PL excited by a probe beam. The PL pump-probe method makes it possible to overcome the limitation of the experimental study of spin dynamics related to finiteness of the electron-hole pair lifetime. At the same time, this method allows one to retain the high sensitivity and spectral selectivity characteristic of the conventional PL techniques. For excitation of the PL we used a tunable Ti:sapphiere laser with the 2-ps pulse duration and the $82-\mathrm{MHz}$ repetition rate.

The structure under study is described in Ref. 14. The lowest electron level in the QDs is close to the Fermi level of the doped substrate that allowed us to control the amount of the resident electrons in the QDs by means of an external voltage. Most measurements were done in a magnetic field parallel to the growth axis of the structure (longitudinal magnetic field) $B=0.1 \mathrm{~T}$. This, according to Ref. 15 , makes it possible to suppress the effect of the nuclear field fluctuations on the electron spin orientation.

As an indication of the resident electron spin orientation, we used the effect of negative circular polarization (NCP) of PL of the charged InP QDs observed when the energy difference of the exciting and detected photons (Stokes shift) exceeds $30 \mathrm{meV}$ [see inset in Fig. 1(a)]. The highest NCP value was obtained in the PL kinetics measured in real time by a streak camera with a Stokes shift of $45 \mathrm{meV}$ [Fig. 1(a)]. The degree of polarization calculated in a standard way, $\rho=\left(I^{+}-I^{-}\right) /\left(I^{+}+I^{-}\right)$, where $I^{+}\left(I^{-}\right)$is the copolarized (crosspolarized) PL intensity, changes its sign in approximately 100 ps after the excitation and then comes to a negative steady-state value further referred to as the NCP amplitude [Fig. 1(b)].

The experiments have shown that the NCP amplitude strongly depends on the bias, $U_{\text {bias, }}$ applied to the sample [right inset in Fig. 1(b)]. The greatest NCP is reached in a narrow bias window near $U_{\text {bias }} \approx-0.1 \mathrm{~V}$ practically coincident with the range where, according to Ref. 14, the trionic quantum beats are observed. So we can conclude that it is the trionic states that are responsible for the arising NCP.

We suppose that the negatively polarized PL is emitted by QDs with parallel electron spins (see left part of the scheme in Fig. 2). ${ }^{16}$ This spin configuration is possible under excitation with a large Stokes shift when the photoexcited and resident electrons occupy different energy states. In these dots, referred to as the $P$-type QDs, the direct relaxation of 

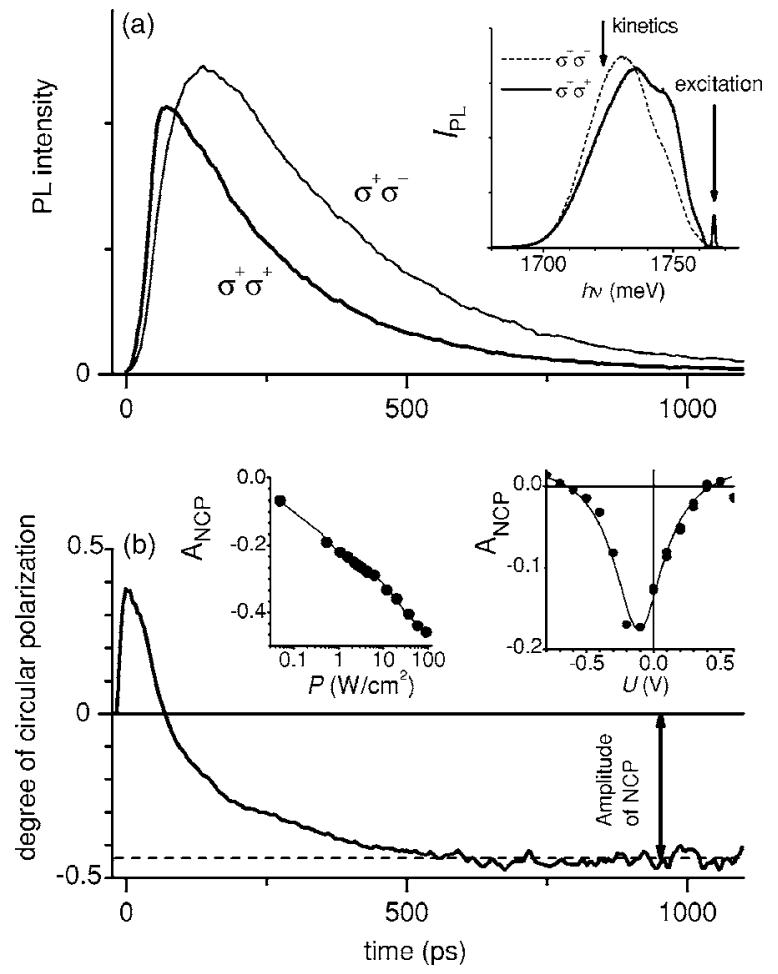

FIG. 1. (a) PL kinetics under strong $\sigma^{+}$excitation in copolarization $\left(\sigma^{+} \sigma^{+}\right)$and cross-polarization $\left(\sigma^{+} \sigma^{-}\right) . P_{\text {ex }}=90 \mathrm{~W} / \mathrm{cm}^{2}, U_{\text {bias }}$ $=-0.1 \mathrm{~V}, T=5 \mathrm{~K}$. The inset shows PL spectra in the copolarization and cross-polarization under excitation at $E_{\text {exc }}=1771 \mathrm{meV}$. (b) Kinetics of the degree of circular polarization. Left inset shows the dependence of the NCP amplitude on the pump power density. Right inset shows the dependence of the NCP amplitude on the bias voltage at weak excitation $P_{\mathrm{ex}} \sim 1 \mathrm{~W} / \mathrm{cm}^{2}$.

the photoexcited electron to the ground state is blocked due to the Pauli principle. However, the combined energy relaxation of the electron and hole with simultaneous flips of their spins (flip-flop process) appears to be allowed in the presence of anisotropic component of the exchange coupling ${ }^{17}$ (see middle part of the scheme). As a result, the electron spins become paired while the hole spin orientation appears to be inverted. In this case, the PL polarization determined by the hole spin becomes negative (see right part of the scheme).

In the QDs with initially antiparallel spins of the photoexcited and resident electrons (the A-type QDs), the exchange coupling is small, and the flip-flop process is not effective. The polarization of the PL of such QDs coincides with that of the excitation, i.e., the degree of polarization is positive. As a result, the contributions to the PL polarization from the $P$-and $A$-type QDs should partially compensate each other, and the total PL should be almost unpolarized if the resident electron spin has no preferential orientation.

If spins of the resident electrons are polarized by the exciting light, ${ }^{18}$ the numbers of the $A$ - and $P$-type QDs become unequal and degree of the PL polarization may be changed. We have detected these changes. As the pump power density was increased, we observed a rapid growth of the NCP with a saturation at about $-50 \%$ [see left inset in Fig. 1(b)]. An evident reason for this growth is the orientation of the resi-

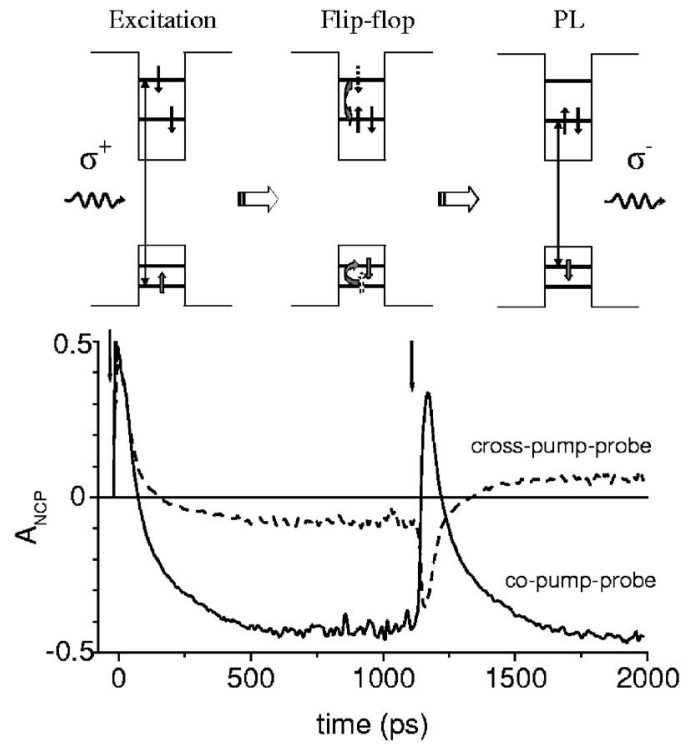

FIG. 2. Kinetics of the degree of circular polarization for the copolarized and cross-polarized pump and probe pulses. Time positions of the pulses are indicated by arrows. The scheme illustrates the model of the NCP formation.

dent electron spins by the exciting beam. Thus, the degree of spin orientation can be found from the measurements of the NCP.

In order to study relaxation of the resident electron spin, we measured kinetics of the PL excited by laser pulses separated in time. For this purpose, the laser beam was split into two beams and one of them was optically delayed (for $\Delta t$ $>1 \mathrm{~ns})$ to avoid any overlap in time of the PL excited by each of the pulses. Polarization of each beam could be varied independently. The results of the experiments for the case of equal intensities of the probe and pump beams are shown in Fig. 2. When the QDs are excited by circularly copolarized beams, a large NCP of both PL pulses is observed. However, in the case of excitation by the cross-polarized beams, the PL polarization virtually vanishes.

These results show that the spin orientation created by the pump pulse affects the polarization of the PL excited by the probe one and vice versa. It is evident that, after recombination of the electron-hole pairs $\left(\tau_{\mathrm{PL}}=250 \mathrm{ps}\right)$, the information about polarization of the excitation can be stored only in the orientation of the resident electron spin. As it is seen from Fig. 2, the NCP amplitude at co-polarized excitation is approximately the same both after the probe and after the pump pulses, in spite of the fact that the former arrives $11 \mathrm{~ns}$ after the latter, while the latter comes $1 \mathrm{~ns}$ after the former. This means that the relaxation time of the electron spins substantially exceeds the laser pulse repetition period $12 \mathrm{~ns}$.

To check the conservation of the spin orientation at much longer times, we have radically increased the time delay between the pump and probe excitation pulses. For this purpose, we selected the laser pulses using acousto-optical modulators $(\mathrm{AOM})$ placed into the pump and probe paths. The repetition period of the pump and probe pulse trains, their duration and the delay were controlled by a functional generator connected with the AOMs. To obtain a noticeable 


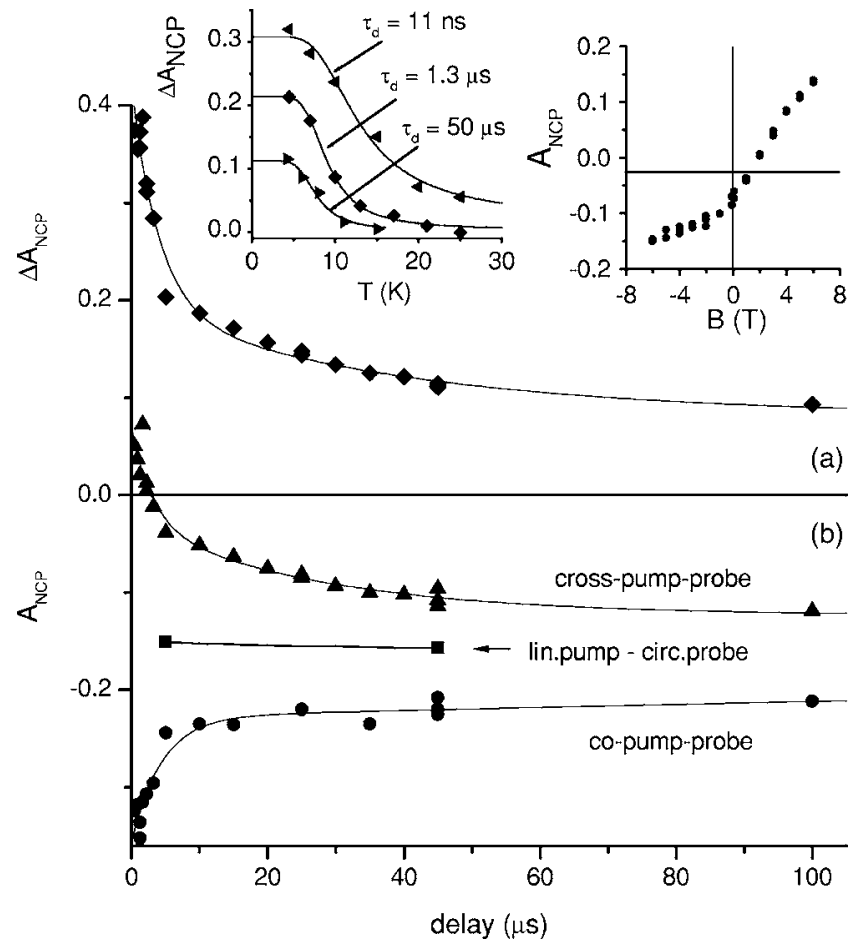

FIG. 3. (a) The time delay dependence of $A_{\mathrm{NCP}}$ for circularly polarized probe with copolarized (circles), cross-polarized (triangles), and linearly (squares) polarized pumps. $T=2 \mathrm{~K}$. (b) The delay dependence of $\Delta A_{\mathrm{NCP}}=A_{\mathrm{NCP}}($ cross $)-A_{\mathrm{NCP}}(\mathrm{co})$ (diamonds) and multiexponential fit (solid line). Left inset: temperature dependence of $\Delta A_{\mathrm{NCP}}$ at different delays $\tau_{d}$ (symbols); solid curves are the fits by the function $f(T)=f_{0}(1+a \beta)^{-1}$, where $\beta=\exp (-\Delta E / k T)$. Activation energy $\Delta E=4 \mathrm{meV}$ for all the curves (Ref. 20). Right inset shows magnetic field dependence of $A_{\mathrm{NCP}}$ under crosspolarized excitation for the 11-ns delay.

spin polarization created by the pump beam, the pump power density was chosen to be several $\mathrm{W} / \mathrm{cm}^{2}$. The intensity of the probe beam was by a factor of 10-20 lower, because only under these conditions the probe pulse train did not erase the spin polarization created by the pump.

We have measured the dependence of the NCP amplitude of the PL excited by the probe beam on time delay between the pump and probe pulse trains [see Fig. 3(a)]. Under linear polarization of the pump beam, the NCP amplitude slightly exceeds $10 \%$ and almost does not depend on the time delay. The circularly polarized pump substantially changes the value of the NCP. These changes are of opposite signs for the copolarized and cross-polarized pumping and are approximately equal in magnitude. Therefore, we attributed the NCP changes to the optical spin orientation rather than to other effects such as, e.g., heating of the structure by the highpower pump. Difference between the NCP amplitudes at the copolarized and cross-polarized pumping can serve as a quantitative characteristics of spin orientation of the resident electrons.

As seen from Fig. 3(b), the spin orientation created by the pump decays in time. Time dependence of the NCP, measured experimentally, cannot be described by a simple exponential function. For the delays of about units of microseconds, one can observe a relatively fast decrease of the orientation and, then, for the time delays exceeding $50 \mu \mathrm{s}$, the orientation comes to a virtually constant level. The reasons for the nonexponential decay of the spin polarization may be related to a spread of the spin relaxation rates in the inhomogeneous ensemble of the QDs caused by the random distribution of uncontrollable paramagnetic defects. The NCP amplitude decreases with temperature and its decay becomes faster. However, even at $T=8 \mathrm{~K}$, the NCP is observable up to time delay $100 \mu$ s. Temperature dependences of the NCP at different delays is shown in the left inset in Fig. 3.

The main conclusion from these experimental results is that the electron spin orientation can be conserved in the ensemble of InP QDs during $100 \mu$ s and more. As it was mentioned above the reason for this spin memory can be not only slow spin relaxation but also the dynamical nuclear polarization conserving the average orientation of the electron spin. The conservation can be realized by the two opposite ways depending on ratio of the electron-nuclear spin exchange rate to the phonon-mediated electron spin relaxation rate.

Fast electron-nuclear spin exchange would result in creation of the common electron-nuclear spin system. Spinphonon interaction for nuclei is so small that the only effective channel of spin losses in the system is the phononmediated relaxation of the electron spin. This channel serves as a "bottleneck" for the spins accumulated in the nuclear reservoir that gives rise to slowing down spin relaxation in the electron-nuclear system.

In the opposite case, when the phonon-mediated electron spin relaxation is faster than the electron-nuclear spin exchange, the electron and nuclear spins can be considered as the independent, though interacting, systems. The light induced electron spin orientation is partially transferred to the nuclear spins causing the dynamic nuclear polarization. An effective magnetic field created by the nuclear polarization splits the electron spin states. At low temperatures, spinphonon relaxation results in freezing out of the electron to the lowest Zeeman level. ${ }^{10}$ Electron polarization created by this way will hold as far as the nuclear field exists.

Quantitative information about possibility of the electron spin freezing in the nuclear field was obtained by measuring the magnetic-field dependence of the NCP in Faraday configuration. The measurements were performed for the crosspolarized excitation by the probe and pump pulses with equal intensities. Under this excitation, neither the electron spin polarization nor the dynamic nuclear spin polarization may appear, and the only source of orientation is the freezing of the electron spin in the external magnetic field. The experiment reveals nearly linear magnetic field dependence of NCP (see right inset in Fig. 3). We may use this dependence to calibrate the rate of the NCP changes due to the freezing effect: $a_{\mathrm{NCP}} \equiv d A_{\mathrm{NCP}} / d B_{\text {ext }} \approx 0.025 \mathrm{~T}^{-1}$.

In the case of the copolarized pump-probe excitation, the electron spin polarization and, as a result, the dynamic nuclear spin polarization may appear. If we assume that the difference between the NCP amplitudes in small external magnetic field for the cross-polarized and copolarized pumping, $\Delta A_{\mathrm{NCP}}=0.35$ (obtained from Fig. 2), is due to establishment of thermal equilibrium in the effective magnetic field of 
nuclei, we can estimate the field $B_{N}=\Delta A_{\mathrm{NCP}} / a_{\mathrm{NCP}}=14 \mathrm{~T}$. This value is larger by approximately three order of magnitude than the nuclear field strength obtained experimentally for the InP QDs. ${ }^{11,19}$ Therefore the freezing effect cannot be responsible for the long-lived electron spin memory in the QDs under study.

So small nuclear field cannot be also responsible for stabilization of the electronic spin due to its coupling to the nuclear spin system by the mechanism mentioned above. Indeed, the nuclear field $B_{N}=0.02 \mathrm{~T}$ (Ref. 11) corresponds to orientation of small fraction of the nuclear spins $(<1 \%)$. If the electron and nuclear spin subsystems are in equilibrium with each other, degree of the electron spin orientation cannot exceed this value. ${ }^{10}$ At the same time, the long-lived component of the degree measured experimentally (see Fig. 3 ) is about $10 \%$. This means that the relaxation rate of the electron spin in the structure under study is really small and quantum information written in such spin system can be conserved for a long time.
In conclusion, our experiments have shown that the quasiresonant excitation of PL of the singly charged InP QDs by a circularly polarized light results in an efficient orientation of the resident electron spins. Decay of the spin orientation is not exponential which is possibly related to the presence of paramagnetic defects in vicinity of the QDs. The long-lived component of the spin orientation is characterized by an effective lifetime exceeding $100 \mu$ s. It is shown that so longlived spin memory arises from the inherently slow electron spin relaxation in the InP QDs rather than due to the nuclear spin polarization.

The authors thank K. V. Kavokin for fruitful discussions, V. S. Zapaskii for critical reading of the manuscript and W. Maruyama for technical assistance in the experiments. This work was supported by Grant-in-Aid for Scientific Research No. 13852003 and No. 16031203 from the MEXT of Japan, by ISTC (Project No. 2679), by INTAS (Project No. 1B 2167), and by RFBR (Project No. 05-02-19913).
*Electronic address: ivan_gnatiev@mail.ru

${ }^{1}$ D. D. Awschalom, D. Loss, and N. Samarth, Semiconductor Spintronics and Quantum Computation (Springer-Verlag, Berlin, 2002).

${ }^{2}$ A. V. Khaetskii and Yu. V. Nazarov, Phys. Rev. B 61, 12639 (2000).

${ }^{3}$ J. M. Kikkawa and D. D. Awschalom, Phys. Rev. Lett. 80, 4313 (1998).

${ }^{4}$ S. Cortez, O. Krebs, S. Laurent, M. Senes, X. Marie, P. Voisin, R. Ferreira, G. Bastard, J-M. Gérard, and T. Amand, Phys. Rev. Lett. 89, 207401 (2002).

${ }^{5}$ R. Hanson, B. Witkamp, L. M. K. Vandersypen, L. H. Willems van Beveren, J. M. Elzerman, and L. P. Kouwenhoven, Phys. Rev. Lett. 91, 196802 (2003).

${ }^{6}$ S. Laurent, O. Krebs, S. Cortez, M. Senes, X. Marie, T. Amand, P. Voisin, and J-M. Gérard, Physica E (Amsterdam) 20, 404 (2004).

${ }^{7}$ M. Kroutvar, Y. Ducommun, D. Heiss, M. Bichler, D. Schuh, G. Abstreiter, and J. J. Finley, Nature (London) 432, 81 (2004).

${ }^{8}$ S. Yu. Verbin, A. Greilich, D. R. Yakovlev, M. Bayer, V. Stavarache, D. Reuter, and A. Wieck, Proceedings of 13th International Symposium "Nanostructures: Physics and Technology", St Petersburg, Russia, 20-25 June 2005 (Ioffe Institute, St. Petersburg, 2005).

${ }^{9}$ J. S. Colton, T. A. Kennedy, A. S. Bracker, and D. Gammon, Phys. Rev. B 69, 121307(R) (2004).

${ }^{10}$ Optical Orientation, edited by B. Meier and B. P. Zakharchenya (North Holland, Amsterdam, 1984).
${ }^{11}$ R. I. Dzhioev, B. P. Zakharchenya, V. L. Korenev, and M. V. Lazarev, Fiz. Tverd. Tela (Leningrad) 41, 2193 (1999) [Phys. Solid State 41, 2014 (1999)].

${ }^{12}$ D. Gammon, Al. L. Efros, T. A. Kennedy, M. Rosen, D. S. Katzer, D. Park, S. W. Brown, V. L. Korenev, and I. A. Merkulov, Phys. Rev. Lett. 86, 5176 (2001).

${ }^{13}$ A. S. Bracker, E. A. Stinaff, D. Gammon, M. E. Ware, J. G. Tischler, A. Shabaev, Al. L. Efros, D. Park, D. Gershoni, V. L. Korenev, and I. A. Merkulov, Phys. Rev. Lett. 94, 047402 (2005).

${ }^{14}$ I. E. Kozin, V. G. Davydov, I. V. Ignatiev, A. V. Kavokin, K. V. Kavokin, G. Malpuech, Hong-Wen Ren, M. Sugisaki, S. Sugou, and Y. Masumoto, Phys. Rev. B 65, 241312(R) (2002).

${ }^{15}$ I. A. Merkulov, Al. L. Efros and M. Rosen, Phys. Rev. B 65, 205309 (2002).

${ }^{16}$ Similar model was considered in Ref. 4.

${ }^{17}$ K. V. Kavokin, Phys. Status Solidi A 195, 592 (2003).

${ }^{18}$ The key process for optical orientation of the electron spins is the relaxation of the hole spins. Mechanisms of the optical orientation of spins are extensively discussed in the literature, see, e.g., Refs. 6 and 10 .

${ }^{19}$ Our experiments also show that the effective nuclear field in the system under study does not exceed few hundredths of $T$. Results of these experiments will be published elsewhere.

${ }^{20}$ Though discussion of the temperature experiments is outside the scope of this paper, we can mention here that the thermally activated process responsible for the NCP decrease is most probably the hole spin relaxation. 